\title{
Restoration of Medical Images using Blind Image Deconvolution based on Ant Colony
}

\author{
Kritika Sharma \\ M.tech Student \\ Computer Science \& Engineering Dept. \\ Rayat Institute of Engineering \& Information \\ Technology, Railmajra \\ Punjab INDIA
}

\author{
Sheetal Kundra \\ Asstt. Prof. \\ Computer Science \& Engineering Dept. \\ Rayat Institute of Engineering \& Information \\ Technology, Railmajra \\ Punjab, INDIA
}

\begin{abstract}
Image restoration is the process of clearing the degraded image to obtain the original image. The main aim of the work is to restore the blurred X-Ray image using Blind Image Restoration. It is very important part of image restoration to recover image without the knowledge of the reason of its degradation. At first, the original X-Ray image is degraded using the Degradation Model. With the addition of blur we get the blurred X-Ray image. In the edges of the blurred X-Ray image, the ringing effect can be detected using Ant Colony Optimization method and then it can be removed before restoration process. An estimate is done about the unknown degradation function and using that an estimate of the original $\mathrm{X}$-Ray image is produced. Ant colony Optimization (ACO) is a nature inspired optimization algorithm that is motivated by the natural foraging behavior of ant species.
\end{abstract}

\section{Keywords}

Blind Image Deconvolution, Ant Colony Optimization, X-Ray Images, MSE, PSNR.

\section{INTRODUCTION}

Restoration of various medical images is of great importance these days. Medical images include images like mammographic images, X-Ray images, ultrasound images and many more. X-ray imaging is a popular and most commonly used method for diagnosing the internal bone structures of the body. It is used to find orthopedic damage, tumors, pneumonias, foreign objects, etc. Unfortunately, $\mathrm{X}$-Ray images appear low image quality caused by fairly low spatial resolution and the presence of noise. X-Ray images also suffer from noise and blur hence they also need restoration in order to remove blur and noise. Hence restoration of X-Ray images is very challenging. We will first degrade the original image using Motion Blur then restore using BID-ACO.

This paper is structured as follows: Section II describes the Blind Image Deconvolution. Section III represents BID based on ACO with algorithms. Section IV describes the Methodology. Section V describes Implementation Results. Section VI Conclusion and Future scope. Section VII describes References.

\section{BLIND IMAGE DECONVOLUTION}

Loss of image quality due to degradation has seriously bad effect on the analyzing and processing of image. Blind restoration of degraded image is usually required to improve the image quality, because of the reason that the degradation is partially or completely unknown in many practical situations. Blind image deconvolution is constantly receiving increasing attention from the academic as well the industrial world due to both its theoretical and practical implications.
Blind Deconvolution seeks to deblur an image without knowing the cause of the blur. Blind Deconvolution is a deconvolution [14] technique that permits recovery of the target scene from a single or set of blurred images in the presence of a poorly determined or unknown point spread function(PSF). A good estimate of the PSF is helpful for quicker convergence but not necessary.

\section{BID BASED ON ACO}

The original image is degraded or blurred using degradation model to produce the blurred image. The blurred image should be an input to the BID algorithm. The result of this algorithm produces the deblurred image which can be compared with our original image. Deblurred image has ringing effect on its edges which can be removed with help of ACO. ACO [12] is nature inspired methodology based on the behavior of real ants and their communication scheme by using pheromone trail A moving ant lays some pheromone on the ground, thus marking the path. With time, pheromone trails evaporate. The collective behavior that emerges from the participating agents is a form of positive feedback where the greater the number of ants that follow a trail, the more attractive that trail becomes. Shorter and thus, favorable paths get marched over faster and receive greater compensation for pheromone evaporation. ACO is used for edge detection in the images so that images can be restored more effectively.

Step 1: Read an X-Ray image $\mathrm{f}(\mathrm{x}, \mathrm{y})$ known as input image.

Step 2: Degrade the input image with a blur function to get a blurred image $\mathrm{g}(\mathrm{x}, \mathrm{y})$ known as degraded image.

Step 3: Then restore the degraded image with Blind Image Deconvolution technique to obtain a restored image.

Step 4: The resultant image of step 3 has ringing effect at its edges which reduces the quality of the image and makes the image unclear.

Step 5: To remove this ringing effect at the edges, apply ACO on the restored image for edge detection.

Step 6: Apply edge taper function to remove ringing effects at the edges detected using ACO.

Step 7: Finally, we get a restored image $\mathrm{f}^{\prime}(\mathrm{x}, \mathrm{y})$.

\section{METHODOLOGY}

This paper aims at studying, analyzing and comparing BID and BID-ACO. For making comparison we will consider different sizes of medical images of .jpg (Joint Photographic Experts Group) format. All the implementation is done in Matlab R2012b. For the implementation of Image restoration algorithms, the main issue is to recover the degraded image to a maximum extent. It is necessary that resultant image obtained after applying a restoration algorithm should be close 
to the original image. In order to enhance its performance, BID is implemented using a Swarm Intelligence Technique known as Ant Colony Optimization (ACO). Here we have some input medical images which are taken for analyzing our results for BID and BID-ACO are shown in Figure 1: The input medical image is degraded with Blur.

Type of Blur: Motion Blur

Parameters of Motion Blur:

\section{Length: 5}

Angle: 5

ACO is used to detect the edges of the images, as the deblured images have ringing effect at its edges The ringing effect can be removed using edge taper function[13] which is used to preprocess our image before passing it to the debluring functions.

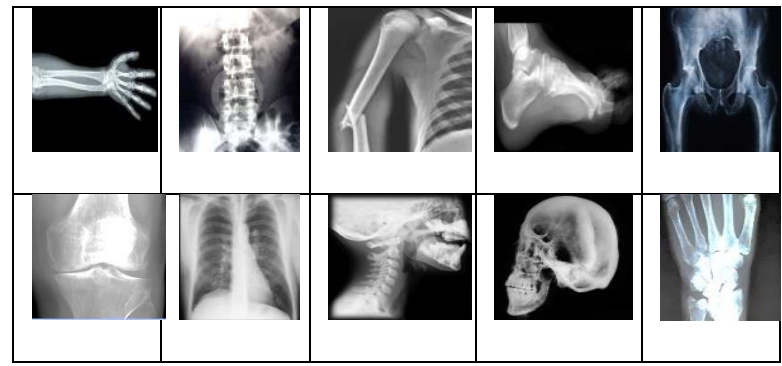

Figure 1: Input X-Ray Images

The overall architecture of the proposed work is described below with the help of Figure 2: It illustrates that Original input X-Ray image will be degraded using some degradation function and motion blur. As result of this degradation, a degraded image will be produced, which can be recovered using BID. Then ACO will be applied to the BID and a resultant image will be produced as a result.

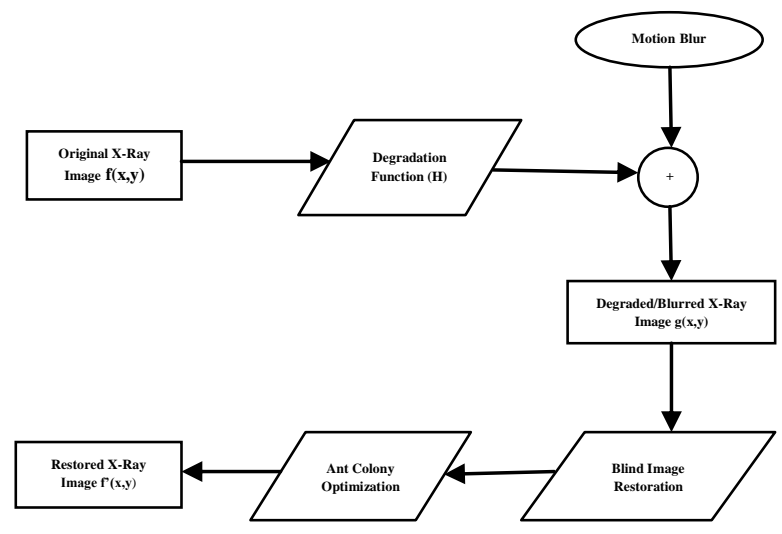

Figure 2: Overall Architecture

\section{IMPLEMENTATION RESULTS}

These images are degraded with degradation function and then restored with BID and BID-ACO. All the results are obtained of all the ten input images but here we have shown two resultant images.
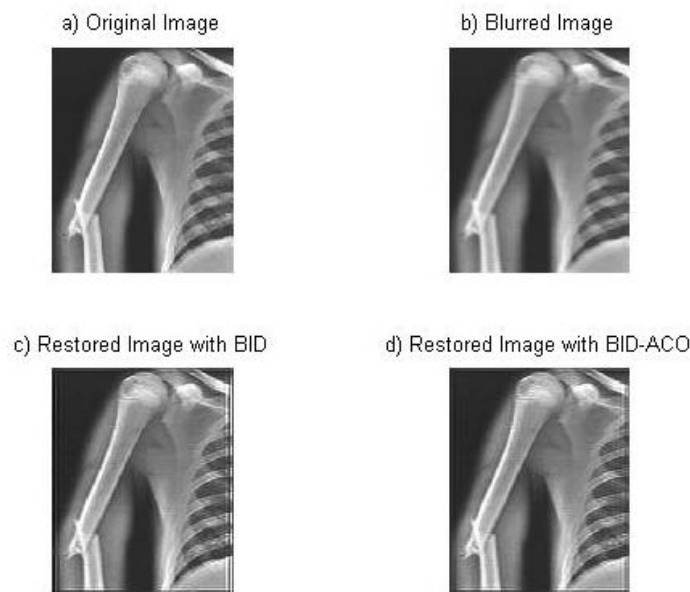

Figure 3: Restoration Results for "brokenbone.jpg" of size " $200 * 248$ "

Figure 3: Illustrates the results of restoration of an image "brokenbone.jpg" of size $200 * 248$ which is degraded by a Motion blur which is having length 5 and angle 5. Image (a) is the original image and image (b) is the blurred image. This blurred image is then tried with BID and BID-ACO for restoration producing resultant images (c) and (d).Conclusion can be drawn from the quality of the resultant images.
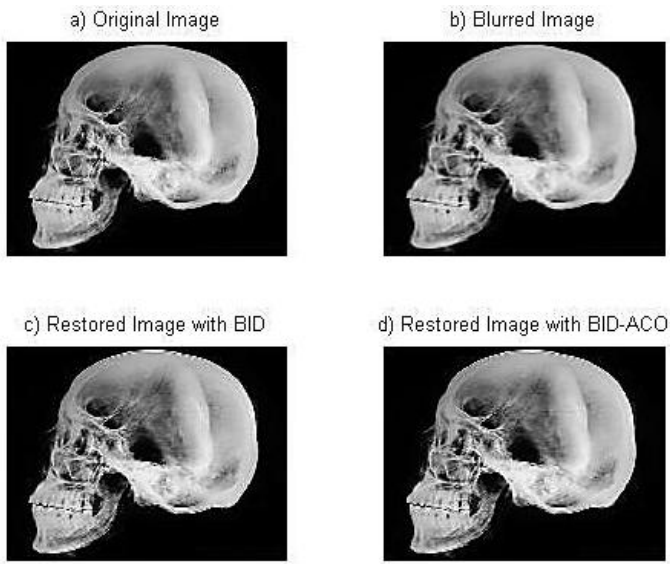

Figure 4: Restoration Results for "skull.jpg" of size "526*401"

Figure 4: Illustrates the results of restoration of an image "skull.jpg" of size $526 * 401$ which is degraded by a Motion blur which is having length 5 and angle 5 . Image (a) is the original image and image (b) is the blurred image. This blurred image is then tried with BID and BID-ACO for restoration producing resultant images (c) and (d). From these images we can conclude about the quality of the resultant images.

This work makes a comparison between BID and BID-ACO on the basis of various performance metrics like MSE, RMSE, PSNR. Following are tabular results obtained after the comparison: 
Table 1: MSE values for BID and BID-ACO

\begin{tabular}{|c|c|c|c|}
\hline Image & Sive & BID & $\mathrm{B} / \mathrm{D}-\mathrm{ACO}$ \\
\hline brokenbone.jpg & $200 \times 248$ & 633.91 & 153.90 \\
\hline arm.jpg & $300 \times 200$ & 540.74 & 121.18 \\
\hline wrist.jpg & $300 \times 250$ & 413.06 & 69.77 \\
\hline neck.jpg & $336 \times 336$ & 174.30 & 13.16 \\
\hline knee.jpg & $400 \times 391$ & 701.38 & 55.28 \\
\hline lungs.jpg & $407 \times 336$ & 599.23 & 366.03 \\
\hline skull.jpg & $526 \times 401$ & 375.40 & 26.44 \\
\hline hip.jpg & $637 \times 480$ & 408.30 & 105.98 \\
\hline backbone.jpg & $683 \times 793$ & 221.59 & 37.97 \\
\hline foot.jpg & $804 \times 449$ & 103.96 & 29.87 \\
\hline
\end{tabular}

Table 1: shows the results of estimation of various medical X-Ray images. Mean Square Error (MSE) values are calculated for BID and BID-ACO algorithm. With the result it proves that BID-ACO is better than BID as it gives lesser MSE value. Lesser the value of MSE more efficient is our algorithm.

Table 2: RMSE values for BID and BID-ACO

\begin{tabular}{lccc}
\hline Image & Size & BID & BID-ACO \\
\hline brokenbone.jpg & $200 \times 248$ & 25.18 & 12.41 \\
\hline arm.jpg & $300 \times 200$ & 23.25 & 11.01 \\
\hline wrist.jpg & $300 \times 250$ & 20.32 & 8.35 \\
\hline neck.jpg & $336 \times 336$ & 13.20 & 3.63 \\
\hline knee.jpg & $400 \times 391$ & 26.48 & 7.44 \\
\hline lungs.jpg & $407 \times 336$ & 24.48 & 19.13 \\
skull.jpg & $526 \times 401$ & 19.38 & 5.14 \\
\hline hip.jpg & $637 \times 480$ & 20.21 & 10.29 \\
\hline backbone.jpg & $683 \times 793$ & 14.89 & 6.16 \\
\hline foot.jpg & $804 \times 449$ & 10.20 & 5.47 \\
\hline
\end{tabular}

Table 2: shows the results of estimation of various medical $\mathrm{X}$-Ray images. Root Mean Square Error(RMSE) values are calculated for BID and BID-ACO algorithm. With the result it proves that BID-ACO is better than BID as it gives lesser RMSE value .Lesser the value of RMSE more efficient is our algorithm.

Table 3: PSNR values for BID and BID-ACO

\begin{tabular}{lccc}
\hline IImage & Size & BID & BID-ACO \\
\hline brokenbone.jpg & $200 \times 248$ & 20.11 & 26.26 \\
\hline arm.jpg & $300 \times 200$ & 20.80 & 27.30 \\
wrist.jpg & $300 \times 250$ & 21.97 & 29.69 \\
neck.jpg & $336 \times 336$ & 25.72 & 36.94 \\
\hline knee.jpg & $400 \times 391$ & 19.67 & 30.70 \\
\hline lungs.jpg & $407 \times 336$ & 20.35 & 22.50 \\
skull.jpg & $526 \times 401$ & 22.39 & 33.91 \\
\hline hip.jpg & $637 \times 480$ & 22.02 & 27.88 \\
\hline backbone.jpg & $683 \times 793$ & 24.68 & 32.34 \\
\hline foot.jpg & $804 \times 449$ & 27.96 & 33.38 \\
\hline
\end{tabular}

Table 3: shows the results of estimation of various medical X-Ray images. Peak Signal to Noise Ratio (PSNR) values are calculated for BID and BID-ACO algorithm .With the result it proves that BID-ACO is better than BID as it gives greater PSNR value .More the value of PSNR more efficient is our algorithm.

A comparison is done on the basis of various performance metrics like MSE (Mean Square Error), RMSE (Root Mean Square Error), PSNR (Peak Signal to Noise Ratio). Graph plots are as follows:

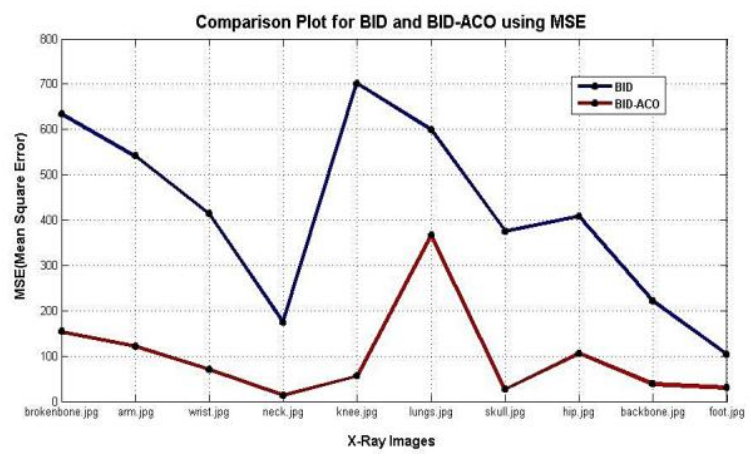

Chart 1: Graph plot for BID and BID-ACO using MSE

$\mathrm{X}$-axis in Graph of Chart 1: Represents various medical images and y-axis shows Mean Square Error (MSE). This comparison plot indicates blue line for BID whereas red line for BID-ACO. BID-ACO produces good results for MSE, lesser the value of MSE more efficient is our algorithm.

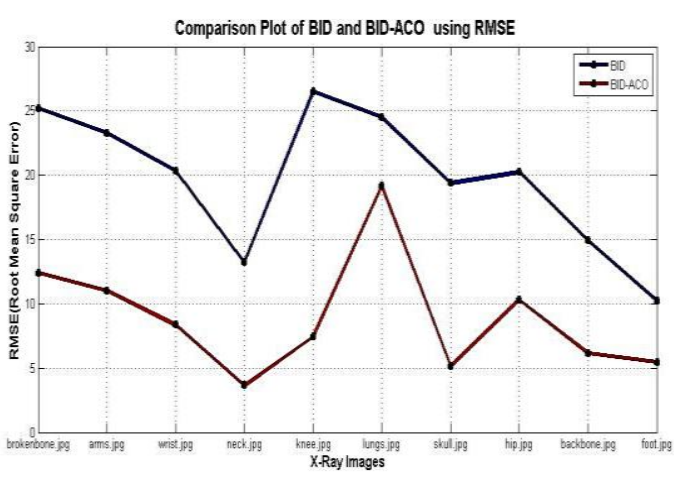

Chart 2: Graph plot for BID and BID-ACO using RMSE

$\mathrm{X}$-axis in Graph of Chart 2: Represents various medical images and y-axis shows Root Mean Square Error (RMSE). This comparison plot indicates blue line for BID whereas red line for BID-ACO.BID-ACO produces good results for RMSE, lesser the value of RMSE more efficient is our algorithm.

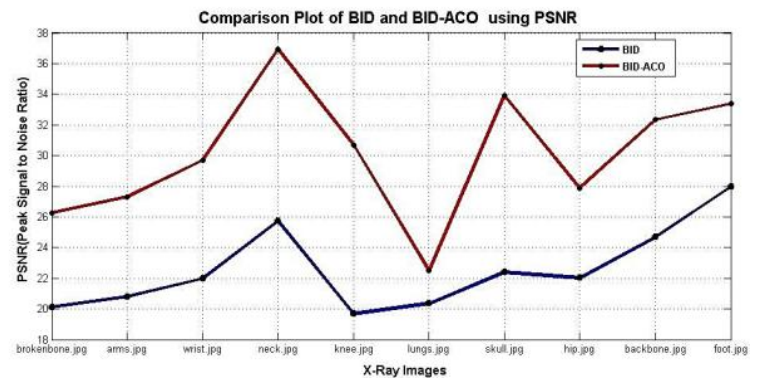

Chart 3: Graph plot for BID and BID-ACO using PSNR 
$\mathrm{X}$-axis in Graph of Chart 3: Represents various medical images and $y$-axis shows Peak Signal To Noise Ratio(PSNR).This comparison plot indicates blue line for BID whereas red line for BID-ACO. BID-ACO produces good results, more the value of PSNR more efficient is our algorithm.

\section{CONCLUSION AND FUTURE WORK}

We have proposed, implemented, and tested the Blind Image Detection (BID) with Ant Colony edge detection based algorithm. The proposed methodology can be used to recover the degraded image to the greater extent. We have implemented BID-ACO to recover the image, overcoming the difficulties and disadvantages of the traditional methods. Integration of BID with ant colony is proposed to detect better edges in medical images and get the better quality resultant image. There are various edge detection methods available to detect an edge of the image such as Sobel, Prewitt, Roberts, and Canny but the edges can be detected effectively using Ant colony Optimization method. As it is clear from the tabular comparison the restoration quality of our method BID-ACO is better than BID .

Further research is needed to explore the ACO in various other fields. Proposed work can be explored with different kinds of images like MRI, Ultrasound, Tumours considering different sizes, types and format of images and different types of blurs and noise then comparing the results.

\section{REFERENCES}

[1] Baterina, A. V. and Oppus, C. 2010 "Image Edge Detection Using Ant Colony Optimization”, International Journal of Circuits, Systems and Signal Processing, pp. 25-33.

[2] Blum, C. and Merkle, D. 2008 "Swarm Intelligence Introduction and Applications". Springer, pp. 43, 58-60.

[3] Campisi, P. and Egiazarian, K. 2006 "Blind image deconvolution theory and applications", CRC Press.

[4] Cao, D.-D and Guo, P. 2005 "Blind image restoration based on wavelet analysis", IEEE, Machine Learning and Cybernetics, pp. 4977-4982.

[5] Corbalan, L.; Massa, G.O.; Russo, C.; Lanzarini, L. and De Giusti, A. 2006 "Image recovery using a new nonlinear adaptive filter based on neural networks", IEEE, Information Technology Interfaces, pp. 355-60.
[6] Kaur, A. and Chopra, V. 2012 "A Comparative Study and Analysis of Image Restoration Techniques Using Different Images Formats", International Journal for Science and Emerging Technologies with Latest Trends, pp. 7-14.

[7] Kaur, A. and Chopra, V. 2012 "Blind Image Deconvolution Technique for Image Restoration using Ant Colony Optimization", International Journal of Computer Applications \& Information Technology, pp. $55-59$

[8] Kundur, D. and Hatzinakos, D. 1998 "A novel blind deconvolution scheme for image restoration using recursive filtering", IEEE Trans. on Signal Processing, vol. 46, no. 2, pp. 375-390.

[9] Kundur, D. and Hatzinakos, D. 1996 "Blind image Deconvolution," IEEE Signal Processing Magazine, pp. 43-64.

[10] Ramya, S. and Mercy Christial, T. 2011 "Restoration of Blurred Images using Blind Deconvolution Algorithm," IEEE, on Emerging Trends in Electrical and Computer Technology (ICETECT), pp. 496-499.

[11] Tian, J.; Yu, W. and Xie, S., 2008 "An Ant Colony Optimization Algorithm for Image Edge Detection", IEEE Congress on Evolutionary Computation, pp. 751756

[12] Yap, K.-H. and Guan, L. 2003 "A computational reinforced learning scheme to blind image deconvolution", IEEE Signal Processing, vol. 51, no. 2, pp. 515-526.

[13] Yap, K.-H., Guan, L. and Liu, W. 2002 “A recursive soft decision approach to blind image deconvolution," IEEE Trans. on Transactions on Evolutionary Computation, vol. 6 , no. 1, pp. 2-15.

[14] Zhao, Z. and Blahut, R.E. 2005 "Blind and nonblind nonnegative impulse response ISI channel demodulation using the Richardson-Lucy Algorithm”, IEEE, Signal Processing and Information Technology, pp. 445-450.

[15] Zhong, C; Fu, J. and Ding, Y. 2011 "Image motion compensation for a certain aviation camera based on Lucy Richardson Algorithm", IEEE, Electronics and Optoelectronics, pp. 41-144. 